\title{
SYMMETRIC NILPOTENT MATRICES WITH MAXIMAL RANK AND A CONJECTURE OF GROTHENDIECK-KOBLITZ
}

\author{
CHING-LI CHAI \\ APPENDIX BY MICHAEL LARSEN \\ (Communicated by Maurice Auslander)
}

\begin{abstract}
All pairs $(p, n)$ such that there exists an $n \times n$ symmetric matrix $A$ with entries in the ring $\mathbb{Z}_{p}$ of $p$-adic integers such that $A^{n}=p \cdot U$ with $U$ invertible in $M_{n \times n}\left(\mathbb{Z}_{p}\right)$ are determined. It is shown that such matrices $A$ can be used to construct examples of deformations of abelian varieties.
\end{abstract}

While contemplating the converse of Grothendieck's specialization theorem for the Newton polygon of $p$-divisible groups and abelian varieties (cf. [G]), I chanced upon the following question in linear algebra.

$Q$. For which integer $n$ and prime number $p$ does there exist an $n \times n$ symmetric matrix $A$ with entries in the ring $\mathbb{Z}_{p}$ of $p$-adic integers such that $A^{n}=p \cdot U$ with $U$ invertible in $M_{n \times n}\left(\mathbb{Z}_{p}\right)$ ?

It turns out that this seemingly innocuous question has an interesting answer, at least when $p$ is an odd prime, which we would like to share with the reader. The connection with Koblitz's conjecture will also be discussed. When $p=2$, $M$. Larsen proved that the answer is always affirmative. It is a pleasure to thank him for writing up his solution as an appendix.

First note that when $p \neq 2$, the question above has an equivalent formulation:

$Q^{\prime}$. For which integer $n$ and prime number $p$ does there exist an $n \times n$ symmetric matrix $A$ with entries in the prime field $\mathbb{F}_{p}$ such that $A$ is nilpotent and has rank $n-1$ ?

The equivalence follows from Hensel's lemma and an easy derivative calculation:

$$
\begin{aligned}
d\left(\operatorname{det}\left(x_{i j}\right)_{1 \leq i, j \leq n}\right) & =\sum_{1 \leq i, j \leq n} A_{i j} d x_{i j} \\
& =\sum_{1 \leq i \leq n} A_{i i} d x_{i i}+2 \sum_{1 \leq i<j \leq n} A_{i j} d x_{i j}
\end{aligned}
$$

Received by the editors July 31, 1990 and, in revised form, February 5, 1992.

1991 Mathematics Subject Classification. Primary 14K10, 14D10.

The author is partially supported by a grant from NSF. 
if we impose the symmetry condition that $x_{i j}=x_{j i} \forall 1 \leq i, j \leq n$.

We shall first work out the geometry of symmetric nilpotent matrices with maximal rank. Since the prime 2 causes problems at several places, a familiar phenomenon, we shall restrict ourselves to fields of characteristic $\neq 2$. Thus we fix a field $k$ with $\operatorname{char}(k) \neq 2$, a positive integer $n$, and let $V=V_{n}$ be the variety over $k$ such that $V(F)=$ the set of all symmetric $n \times n$ matrices of rank $n-1$ with entries in $F$, for any field $F$ over $k$. The above calculation says that $V$ is smooth over $k$. $V$ has lots of symmetries: the orthogonal group $\mathrm{O}_{n}$ (for the standard form) operates on $V$ by $g: A \mapsto g A g^{-1}=g A^{t} g, \forall g \in \mathrm{O}_{n}$, $\forall A \in V$.

Examples. (i) When $n=2$,

$$
V_{2}=\left\{\left[\begin{array}{cc}
a & b \\
b & -a
\end{array}\right]: a^{2}+b^{2}=0, a \neq 0, b \neq 0\right\}
$$

hence, $V\left(\mathbb{F}_{p}\right) \neq \varnothing$ iff $p \equiv 1(\bmod 4)$.

(ii) When $n=3$, the rational point

$$
\left[\begin{array}{ccc}
1 & \sqrt{-1} & 1 \\
\sqrt{-1} & -1 & \sqrt{-1} \\
1 & \sqrt{-1} & 0
\end{array}\right]
$$

in $V_{3}(\mathbb{Q}(\sqrt{-1}))$ shows that $V_{3}\left(\mathbb{F}_{p}\right) \neq \varnothing$ if $p \equiv 1(\bmod 4)$.

(iii) When $n=3$ and $p=3, V_{3}\left(\mathbb{F}_{3}\right) \neq \varnothing$ since

$$
\left[\begin{array}{ccc}
0 & 1 & -1 \\
1 & -1 & 0 \\
-1 & 0 & 1
\end{array}\right]
$$

is a rational point in $V_{3}\left(\mathbb{F}_{3}\right)$.

The following lemma is very useful.

Lemma. For any field $F$ over $k$ and any $A$ in $V(F)$, the commutator of $A$ inside $M_{n \times n}(F)$ is just $F[A]\left(=F+F A+F A^{2}+\cdots+F A^{n-1}\right)$, which as an $F$-algebra is isomorphic to $F[x] /\left(x^{n}\right)$.

Proof. We shall prove this statement for any nilpotent matrix $B$ of maximal rank. Hence we can assume that $B$ is already in the canonical form with 1 's below the diagonal. The proof is finished by a direct computation.

Psychologically the first thing we want to know about $V$ is that it is not empty. Thus let $N$ be the nilpotent $n \times n$ matrix with 1's below the diagonal; we want to find some $g \in \mathrm{GL}(\bar{k})$ such that $g N g^{-1}$ is symmetric. Clearly $g N g^{-1}={ }^{t} g^{-1 t} N^{t} g \Leftrightarrow{ }^{t} g g N={ }^{t} N^{t} g g$. We know that (by Witt's theorem) $g \mapsto{ }^{t} g g$ establishes an isomorphism $\mathrm{O}_{n} \backslash \mathrm{GL}_{n} \approx Z_{n}$ since $\operatorname{char}(k) \neq 2$, where $Z_{n}$ is the variety of all invertible symmetric $n \times n$ matrices. Hence we are reduced to finding a symmetric invertible matrix $X$ such that $X N={ }^{t} N X$. By explicit computation, the necessary and sufficient condition for a symmetric 
matrix $Y$ to satisfy $Y N={ }^{t} N Y$ is that $Y$ has the form

$$
\left[\begin{array}{ccccccc}
a_{n} & a_{n-1} & \cdot & \cdot & \cdot & a_{2} & a_{1} \\
a_{n-1} & a_{n-2} & \cdot & \cdot & a_{2} & a_{1} & 0 \\
a_{n-2} & \cdot & \cdot & a_{2} & a_{1} & 0 & 0 \\
\cdot & \cdot & \cdot & \cdot & \cdot & \cdot & \cdot \\
a_{2} & a_{1} & 0 & \cdot & \cdot & \cdot & 0 \\
a_{1} & 0 & 0 & \cdot & \cdot & \cdot & 0
\end{array}\right]
$$

and the condition for such a $Y$ to be invertible is that the antidiagonal entries (all equal to $a_{1}$ ) are invertible. In particular, such $Y$ 's surely exist. Note that the above analysis also gives an explicit way to construct points in $V$ : one just has to extract a "square root" for an invertible $Y$ as above.

Next we show that $\mathrm{O}_{n}$ operates transitively on $V$. Thus let $A_{1}, A_{2}$ be elements of $V(\bar{k})$; there exists (by the theory of canonical forms) an element $g \in \mathrm{GL}_{n}(\bar{k})$ such that $g A_{1} g^{-1}=A_{2}$, and we want to show that there exists a $g_{1} \in \mathrm{O}_{n}(\bar{k})$ such that $g_{1} A_{1} g_{1}^{-1}=A_{2}$. We have $g A_{1} g^{-1}=A_{2} \Rightarrow g A_{1}=A_{2} g$, $A_{1}{ }^{t} g={ }^{t} g A_{2} \Rightarrow{ }^{t} g g A_{1}={ }^{t} g A_{2} g=A_{1}{ }^{t} g g$; hence by the lemma we know that ${ }^{t} g g \in \bar{k}\left[A_{1}\right]^{\times}$. Since $\operatorname{char}(k) \neq 2$ and $A_{1}$ is nilpotent, there exists an $h \in$ $\bar{k}\left[A_{1}\right]^{\times}$such that ${ }^{t} g g=h^{2}$. (A trivial case of Hensel's lemma, since $\bar{k}\left[A_{1}\right] \approx$ $\bar{k}[x] /\left(x^{n}\right)$.) Let $g_{1}=g h^{-1}$. We have ${ }^{t} g_{1} g_{1}=h^{-1} t g g h^{-1}=h^{-1} h^{2} h^{-1}=1$ since $h$ is symmetric (as in $A_{1}$ ); hence, $g_{1} \in \mathrm{O}_{n}(\bar{k})$, and clearly $g_{1} A_{1} g_{1}^{-1}=$ $g A_{1} g^{-1}=A_{2}$.

Finally let us pick and fix $A_{0} \in V(\bar{k})$. We want to find its stabilizer in $\mathrm{O}_{n}$. Suppose $g A_{0} g^{-1}=A_{0}$. By the lemma we know that $g \in \bar{k}\left[A_{0}\right]^{\times}$; hence, $1={ }^{t} g g=g^{2}$. Since $\operatorname{char}(k) \neq 2$, we conclude that $g= \pm 1$ again because $\bar{k}\left[A_{0}\right] \approx \bar{k}[x] /\left(x^{n}\right)$. So we have proved:

Proposition 1. $V_{n}$ is a principal homogeneous space for $\mathrm{PO}_{n}=\mathrm{O}_{n} /\{ \pm 1\}$ over $k$. Therefore $V_{n}$ is a principal homogeneous space for $\mathrm{SO}_{n}$ if $n$ is odd, while $V_{n}$ has two geometric components if $n$ is even.

Now we take $k=\mathbb{F}_{p} \quad(p \neq 2)$. If $n$ is odd, we conclude that $V$ has an $\mathbb{F}_{p}$-rational point by Lang's theorem. (Every principal homogeneous space for a connected algebraic group over a finite field is trivial, see [La].) If $n=2 m$ is even, again by Lang's theorem we see that $V\left(\mathbb{F}_{p}\right) \neq \varnothing$ iff both geometric components of $V$ are defined over $\mathbb{F}_{p}$; hence, we must compute the Galois action on the geometric components of $V$. This can be done by constructing rational points over a quadratic extension of $k$, as explained in the first part of the proof of Proposition 1. If we take the $Y$ there to be

$$
Y_{0}=\left[\begin{array}{cccccc}
0 & 0 & \cdots & 0 & 0 & 2 \\
0 & 0 & \cdots & 0 & 2 & 0 \\
\vdots & \vdots & & \vdots & \vdots & \vdots \\
2 & 0 & \cdots & 0 & 0 & 0
\end{array}\right]
$$

we have to find a $g_{0} \in \mathrm{GL}_{n}$ such that ${ }^{t} g_{0} g_{0}=Y_{0}$. Any such $g_{0}$ produces a point $g_{0} N g_{0}^{-1} \in V(\bar{K})$ by the first paragraph in the proof of Proposition 1 .

Taking $k$ to be $\mathbb{Q}$, we have

$$
\left[\begin{array}{cc}
1 & -\sqrt{-1} \\
1 & \sqrt{-1}
\end{array}\right]\left[\begin{array}{cc}
1 & 1 \\
-\sqrt{-1} & \sqrt{-1}
\end{array}\right]=\left[\begin{array}{ll}
0 & 2 \\
2 & 0
\end{array}\right]
$$


So we can take $g_{0}$ to be

$$
\left[\begin{array}{cc}
I_{m} & J_{m} \\
-\sqrt{-1} J_{m} & \sqrt{-1} I_{m}
\end{array}\right]
$$

where $J_{m}$ denotes the $m \times m$ matrix with 1's on the antidiagonal and 0's elsewhere and $g_{0} N g_{0}^{-1} \in V(\mathbb{Q}(\sqrt{-1}))$. If $\operatorname{Gal}(\mathbb{Q}(\sqrt{-1}) / \mathbb{Q})=\{\mathrm{id}, \tau\}$, then

$$
g_{0}^{\tau}=\left[\begin{array}{cc}
I_{m} & 0 \\
0 & -I_{m}
\end{array}\right] \cdot g_{0}
$$

Since

$$
\operatorname{det}\left(\left[\begin{array}{cc}
I_{m} & 0 \\
0 & -I_{m}
\end{array}\right]\right)=(-1)^{m},
$$

we see that $\tau$ operates on the two geometric components of $V / \mathbb{Q}$ by $(-1)^{m}$, i.e., $\tau$ stabilizes both geometric components if $n \equiv 0(\bmod 4)$, and $\tau$ interchanges them if $n \equiv 2(\bmod 4)$. From the splitting behavior of primes in $\mathbb{Q}(\sqrt{-1}) / \mathbb{Q}$, we conclude:

Proposition 2. Suppose $n$ is even and $p$ is an odd prime number. Then

(i) if $n \equiv 0(\bmod 4)$ or $p \equiv 1(\bmod 4)$, then each geometric component of $V_{n / \mathbb{F}_{p}}$ is defined over $\mathbb{F}_{p}$ and is a principal homogeneous space under $\mathrm{PSO}_{n}=$ $\mathrm{SO}_{n} /\{ \pm 1\}$

(ii) if $n \equiv 2(\bmod 4)$ and $p \equiv 3(\bmod 4)$, then the Frobenius interchanges the two geometric components of $V_{n / \mathbb{F}_{p}}$.

We summarize our results as

Theorem. Let $p$ be an odd prime number and $n$ a positive integer. Then $V_{n}\left(\mathbb{F}_{p}\right)=\varnothing$ iff $p \equiv 3(\bmod 4)$ and $n \equiv 2(\bmod 4)$. Therefore there exists a symmetric $n \times n$ matrix with entries in $\mathbb{Z}_{p}$ whose nth power is equal to $p$ times a unit in $M_{n \times n}\left(\mathbb{Z}_{p}\right)$ iff $p \equiv 1(\bmod 4)$ or $n \equiv 0,1$, or $3(\bmod 4)$.

Remark. Let $W=W_{n}$ be the variety of all symmetric $n \times n$ mattices. $\mathrm{O}_{n}$ operates on $W$ and $V$ is contained in the null cone. As the referee pointed out, the ring of invariants of $\mathrm{O}_{n}$ operating on $W$ is a polynomial ring with the coefficients of the characteristic polynomials as generators, at least in characteristic 0 . This implies that the null cone has a beautiful structure.

We now turn to discuss the connection with a conjecture of GrothendieckKoblitz (cf. [Ko]), which states that if $N P_{1}, \ldots, N P_{m}$ is a sequence of Newton polygons with the same end points such that $N P_{i+1}$ lies above $N P_{i}$ for each $i$, then this sequence can be realized by successive specialization of principally polarized varieties in characteristic $p>0$. To the best of my knowledge, this conjecture is still open and so is the case for Barsotti-Tate groups. ${ }^{1}$ Here we shall give some examples such that all slopes of $N P_{m}$ are $\frac{1}{2}$ (this is not a restriction because this $N P_{m}$ is the highest possible Newton polygon), by deforming a product of supersingular elliptic curves. We shall not try to state our result in the ultimate generality; rather we only present our calculation as a way to construct some example of deformations.

Let $k$ be an algebraically closed field of characteristic $p>0$, and let $M_{0}$ be the Dieudonné module of a product of $r$ copies of supersingular elliptic curves.

\footnotetext{
${ }^{1}$ Shortly after this paper was submitted, this conjecture was proved by F. Oort. A preliminary note of his important result is available and titled Moduli of abelian varieties and Newton polygons.
} 
In other words, $M_{0}$ has a free $W(k)$ basis $x_{1}, \ldots, x_{r}, y_{1}, \ldots, y_{r}$, and the Frobenius and Verschibung send $x_{i}$ to $y_{i}: F x_{i}=y_{i}=V x_{i}, 1 \leq i \leq r$. The product polarization corresponds to the symplectic pairing having $x_{1}, \ldots, x_{r}$, $y_{1}, \ldots, y_{r}$ as a symplectic basis: $\left\langle x_{i}, y_{j}\right\rangle=\delta_{i j}$. Using the Cartier-Dieudonné theory, a general deformation of $M_{0}$ over a complete local ring $R$ over $k$ can be given as follows:

$$
F x_{i}=\sum T_{i j} x_{j}+y_{i}, \quad V x_{i}=y_{i}, \quad 1 \leq i \leq r,
$$

where $T_{i j}=\left[t_{i j}\right]=\left(t_{i j}, 0,0, \ldots\right)$ is the Teichmüller representative of $t_{i j}$ in the ring of infinite Witt vectors $W(R), t_{i j} \in R, 1 \leq i, j \leq r$. If we write $F$ in block form, its entries are $T, p I_{r}, I_{r}, 0$, where $T=\left(T_{i j}\right)$. If we take polarization into account and want the product polarization to extend to the deformation, we must require that $t_{i j}=t_{j i}, 1 \leq i, j \leq r$. In practice, we shall choose $R$ to be a formal power series ring (possibly with several variables), and we want to estimate the slope of the deformation over the fraction field of $R$. One way to do this is to use the result in $[\mathrm{Ka}]$, which requires some information about iteration of Frobenius.

We shall write the $n$th iteration $F^{n}$ of $F$ in block form with entries $A^{(n)}$, $B^{(n)}, C^{(n)}, D^{(n)}$. Let us first see some examples:

$$
\begin{aligned}
& n=2: A^{(2)}=T T^{\sigma}+p, B^{(2)}=p T, C^{(2)}=T^{\sigma}, D^{(2)}=p \text {, where } \sigma \text { denotes } \\
& \text { the Frobenius automorphism on } W(R) \text {. } \\
& n=3: A^{(3)}=T T^{\sigma} T^{\sigma^{2}}+p T^{\sigma^{2}}+p T, B^{(3)}=p T T^{\sigma}+p^{2} \text {, } \\
& C^{(3)}=T^{\sigma} T^{\sigma^{2}}+p, D^{(3)}=p T^{\sigma} \\
& n=4: A^{(4)}=T T^{\sigma} T^{\sigma^{2}} T^{\sigma^{3}}+p T^{\sigma^{2}} T^{\sigma^{3}}+p T T^{\sigma^{3}}+p T T^{\sigma}+p^{2} \text {, } \\
& B^{(4)}=p T T^{\sigma} T^{\sigma^{2}}+p^{2} T^{\sigma^{2}}+p^{2} T, \\
& C^{(4)}=T^{\sigma} T^{\sigma^{2}} T^{\sigma^{3}}+p T^{\sigma^{3}}+p T^{\sigma^{3}}, D^{(4)}=p T^{\sigma} T^{\sigma^{2}}+p^{2} \text {. } \\
& n=5: A^{(5)}=T T^{\sigma} T^{\sigma^{2}} T^{\sigma^{3}} T^{\sigma^{4}}+p T^{\sigma^{2}} T^{\sigma^{3}} T^{\sigma^{4}}+p T T^{\sigma^{3}} T^{\sigma^{4}}+p T T^{\sigma} T^{\sigma^{4}} \\
& +p T^{\sigma^{2}} T^{\sigma^{3}} T^{\sigma^{4}}+p^{2} T^{\sigma^{4}}+p^{2} T^{\sigma^{2}}+p^{2} T, \\
& B^{(5)}=p T T^{\sigma} T^{\sigma^{2}} T^{\sigma^{3}}+p^{2} T^{\sigma^{2}} T^{\sigma^{3}}+p^{2} T T^{\sigma^{3}}+p^{2} T T^{\sigma}+p^{3} \text {, } \\
& C^{(5)}=T^{\sigma} T^{\sigma^{2}} T^{\sigma^{3}} T^{\sigma^{4}}+p T^{\sigma^{3}} T^{\sigma^{4}}+p T^{\sigma} T^{\sigma^{4}}+p T^{\sigma} T^{\sigma^{2}}+p^{2} \text {, } \\
& D^{(5)}=p T^{\sigma} T^{\sigma^{2}} T^{\sigma^{3}}+p^{2} T^{\sigma^{3}}+p^{2} T^{\sigma} \text {. } \\
& n=6: A^{(6)}=T T^{\sigma} T^{\sigma^{2}} T^{\sigma^{3}} T^{\sigma^{5}}+p T^{\sigma^{2}} T^{\sigma^{3}} T^{\sigma^{4}} T^{\sigma^{5}}+p T T^{\sigma^{3}} T^{\sigma^{4}} T^{\sigma^{5}} \\
& +p T T^{\sigma} T^{\sigma^{4}} T^{\sigma^{5}}+p T T^{\sigma} T^{\sigma^{2}} T^{\sigma^{5}}+p T T^{\sigma} T^{\sigma^{2}} T^{\sigma^{3}}+p^{2} T^{\sigma^{4}} T^{\sigma^{5}} \\
& +p^{2} T^{\sigma^{2}} T^{\sigma^{5}}+p^{2} T T^{\sigma^{5}}+p^{2} T^{\sigma^{2}} T^{\sigma^{3}}+p^{2} T T^{\sigma}+p^{3} \text {, } \\
& B^{(6)}=p T T^{\sigma} T^{\sigma^{2}} T^{\sigma^{3}} T^{\sigma^{4}}+p^{2} T^{\sigma^{2}} T^{\sigma^{3}} T^{\sigma^{4}}+p^{2} T T^{\sigma^{3}} T^{\sigma^{4}}+p^{2} T T^{\sigma} T^{\sigma^{4}} \\
& +p^{2} T T^{\sigma} T^{\sigma^{2}}+p^{3} T^{\sigma^{4}}+p^{3} T^{\sigma^{2}}+p^{3} T \text {, } \\
& C^{(6)}=T^{\sigma} T^{\sigma^{2}} T^{\sigma^{3}} T^{\sigma^{4}} T^{\sigma^{5}}+p T^{\sigma^{3}} T^{\sigma^{4}} T^{\sigma^{5}}+p T^{\sigma} T^{\sigma^{4}} T^{\sigma^{5}}+p T^{\sigma} T^{\sigma^{2}} T^{\sigma^{5}} \\
& +p T^{\sigma} T^{\sigma^{2}} T^{\sigma^{3}}+p^{2} T^{\sigma^{5}}+p^{2} T^{\sigma^{3}}+p^{2} T^{\sigma}, \\
& D^{(6)}=p T^{\sigma} T^{\sigma^{2}} T^{\sigma^{3}} T^{\sigma^{4}}+p^{2} T^{\sigma^{3}} T^{\sigma^{4}}+p^{2} T^{\sigma} T^{\sigma^{4}}+p^{2} T^{\sigma} T^{\sigma^{2}}+p^{3} \text {. }
\end{aligned}
$$


In general we have

$$
\begin{aligned}
A^{(n)}= & T T^{\sigma} T^{\sigma^{2}} \cdots T \sigma^{n-1}+p \cdot \sum \prod \text { (drop a pair of consecutive terms) } \\
& +p^{2} \cdot \sum \prod(\text { drop two pairs of consecutive terms })+\cdots, \\
B^{(n)}= & p T T^{\sigma} T^{\sigma^{2}} \cdots T^{\sigma^{n-2}}+p^{2} \cdot \sum \prod(\text { drop a pair of consecutive terms) } \\
& +p^{3} \cdot \sum \prod(\text { drop two pairs of consecutive terms })+\cdots, \\
C^{(n)}= & T^{\sigma} T^{\sigma^{2}} \cdots T^{\sigma^{n-1}}+p \cdot \sum \prod \text { (drop a pair of consecutive terms) } \\
& +p^{2} \cdot \sum \prod(\text { drop two pairs of consecutive terms })+\cdots \\
D^{(n)}= & p T^{\sigma} T^{\sigma^{2}} \cdots T^{\sigma^{n-2}}+p^{2} \cdot \sum \prod \text { (drop a pair of consecutive terms) } \\
& +p^{3} \cdot \sum \prod(\text { drop two pairs of consecutive terms })+\cdots
\end{aligned}
$$

If we take $R=k[t], T=[t] \cdot A$ where $A \in M_{r \times r}\left(\mathbb{Z}_{p}\right)$ and $A^{r}=p^{s} \cdot$ (unit), $2 s<r$, then any product of $m$ terms of the form $T^{\sigma^{i}}$ is always of the form $p^{[m / r] s}$. (a matrix not congruent to $0 \bmod p$ ). Note that such $A$ exists: start with a cyclic permutation matrix, replace the first $s$ l's by $p$. Moreover since $[t]^{\sigma^{i}}=\left[t^{p^{i}}\right]$, there is no cancellation. Therefore $F^{n}=p^{[n / r] s}$. (a matrix whose determinant is not congruent to $0 \bmod p$ ). Thus by Katz's "basic slope estimate" [Ka, 1.4.3, p. 125], we see that the first $r$ slopes are all equal to $s / r$; therefore, the last $r$ slopes are all equal to $(r-s) / r$. This argument also works for higher-dimensional base rings $R$ : take $s<r / 2, U \in M_{r \times r}\left(\mathbb{Z}_{p}\right)$, $U^{r}=p \cdot$ (unit), and $T=\left[t_{0}\right] \cdot I_{r}+\left[t_{1}\right] \cdot U+\left[t_{2}\right] \cdot U^{2}+\cdots+\left[t_{s}\right] \cdot U^{s}$. This produces a sequence of Newton polygons $N P_{0}, N P_{1}, \ldots, N P_{s}, N P_{s+1}$ such that $N P_{i}$ has slopes $i / r$ and $(t-s) / r$ with multiplicity $r$ if $0 \leq i \leq s$ and $N P_{s+1}$ has slope $1 / 2$ and multiplicity $2 r$. With polarization, we need a symmetric $U \in M_{r \times r}\left(\mathbb{Z}_{p}\right)$ such that $U^{r}=p \cdot($ unit $)$. This is possible if $p \equiv 1(\bmod 4)$, or $r \equiv 0,1,3(\bmod 4)$ and $p>2$. Thus we have shown

Proposition 3. Let $r, s$ be positive integers such that $2 s<r$. Let $N P_{0}$, $N P_{1}, \ldots, N P_{s}, N P_{s+1}$ be the Newton polygons connecting $(0,0)$ to $(2 r, r)$ such that $N P_{i}$ has slopes $i / r$ and $(t-s) / r$ with multiplicity $r$ for $0 \leq i \leq s$ and $N P_{s+1}$ has slope $1 / 2$ and multiplicity $2 r$.

(a) $N P_{0}, N P_{1}, \ldots, N P_{s}, N P_{s+1}$ can always be realized by a Barsotti-Tate group over $k\left[\left[t_{0}, t_{1}, \ldots, t_{s}\right]\right]$.

(b) Assume either $p \equiv 1(\bmod 4)$, or $r \equiv 0,1,3(\bmod 4)$ and $p>2$. Then $N P_{0}, N P_{1}, \ldots, N P_{s}, N P_{s+1}$ can be realized by a principally polarized abelian scheme over $k\left[\left[t_{0}, t_{1}, \ldots, t_{s}\right]\right]$.

\section{APPENDIX: A 2-ADIC LIFTING PROBlem BY MiChael LARSEN ${ }^{2}$}

This appendix gives, for all $n \in N$, an affirmative answer to the question of whether there exists a symmetric matrix $A$ in $M_{n \times n}\left(\mathbf{Z}_{2}\right)$ with $A^{n}$ equal to $2 U, U$ invertible.

\footnotetext{
${ }^{2}$ Michael Larsen was supported by NSF Grant No. DMS-8807203. He is affiliated with the University of Pennsylvania, Philadelphia, Pennsylvania 19104.
} 
We begin by constructing, for each $n$, an $n \times n$ symmetric matrix with entries in $\mathbf{F}_{2}$, nilpotent of rank $n-1$. For $n$ fixed, define $e_{i, j}$ for $1 \leq i, j \leq n$ to be the matrix with $(i, j)$ th entry 1 and all other entries 0 . For $i$ or $j$ out of range, let $e_{i, j}=0$. Let $N=\sum_{i=1}^{n} e_{i, i+1}$ denote the standard nilpotent matrix of rank $n-1$. Over $\mathbf{F}_{2}$,

$$
(I+N)^{-1}=I+N+N^{2}+\cdots+N^{n-1} .
$$

Define the permutation $\sigma:\{1,2, \ldots, n\} \rightarrow\{1,2, \ldots, n\}$ as follows:

$$
\sigma(k)= \begin{cases}2 k & \text { if } k \leq n / 2, \\ 2(n-k)+1 & \text { otherwise }\end{cases}
$$

Let $P_{\sigma}$ denote the corresponding permutation matrix and

$$
M=(I+N)^{-1} P_{\sigma}^{-1} N P_{\sigma}(I+N)=\sum_{1 \leq i \leq j \leq n} e_{i, j} \sum_{i=1}^{n-1} e_{\sigma(i), \sigma(i+1)} \sum_{i=1}^{n}\left(e_{i, i}+e_{i, i+1}\right) .
$$

Evidently $M$ is nilpotent of rank $n-1$; we claim that it is symmetric. The cases $n$ even and $n$ odd must be considered separately.

Suppose $n=2 m$. Then

$$
\sum_{i=1}^{n-1} e_{\sigma(i), \sigma(i+1)}=\sum_{i=1}^{m-1} e_{2 i, 2 i+2}+\sum_{i=1}^{m-1} e_{2 i+1,2 i-1}+e_{2 m, 2 m-1} .
$$

As $e_{i, j} e_{k, l}=\delta_{j, k} e_{i, l}$,

$$
\begin{aligned}
M= & \sum_{j=1}^{m-1} \sum_{i=1}^{2 j} e_{i, 2 j+2}+\sum_{j=1}^{m-2} \sum_{i=1}^{2 j} e_{i, 2 j+3}+\sum_{j=1}^{m-1} \sum_{i=1}^{2 j+1} e_{i, 2 j-1} \\
& +\sum_{j=1}^{m-1} \sum_{i=1}^{2 j+1} e_{i, 2 j}+\sum_{i=1}^{2 m} e_{i, 2 m-1}+\sum_{i=1}^{2 m} e_{i, 2 m}
\end{aligned}
$$

Combining the third and fifth terms and likewise the fourth and sixth terms, we get

$$
\sum_{j=1}^{m-1} \sum_{i=1}^{2 j} e_{i, 2 j+2}+\sum_{j=1}^{m-2} \sum_{i=1}^{2 j} e_{i, 2 j+3}+\sum_{j=1}^{m} \sum_{i=1}^{2 j+1} e_{i, 2 j-1}+\sum_{j=1}^{m} \sum_{i=1}^{2 j+1} e_{i, 2 j}
$$

(harmlessly) extending the ranges of summation,

$$
\begin{aligned}
& \sum_{j=0}^{m} \sum_{i=1}^{2 j} e_{i, 2 j+2}+\sum_{j=0}^{m} \sum_{i=1}^{2 j} e_{i, 2 j+3}+\sum_{j=1}^{m} \sum_{i=1}^{2 j+1} e_{i, 2 j-1}+\sum_{j=1}^{m} \sum_{i=1}^{2 j+1} e_{i, 2 j} \\
& =\sum_{k \text { even }}\left(\sum_{i=1}^{k-2} e_{i, k}+\sum_{i=1}^{k+1} e_{i, k}\right)+\sum_{k \text { odd }}\left(\sum_{i=1}^{k-3} e_{i, k}+\sum_{i=1}^{k+2} e_{i, k}\right) \\
& =\sum_{k \text { even }} \sum_{i=k-1}^{k+1} e_{i, k}+\sum_{k \text { odd }} \sum_{i=k-2}^{k+2} e_{i, k} .
\end{aligned}
$$


The right-hand side is evidently symmetric; the matrix looks like

$$
M_{2 m}=\left(\begin{array}{ccccccccc}
1 & 1 & 1 & 0 & 0 & \ldots & 0 & 0 & 0 \\
1 & 1 & 1 & 0 & 0 & \ldots & 0 & 0 & 0 \\
1 & 1 & 1 & 1 & 1 & \ldots & 0 & 0 & 0 \\
0 & 0 & 1 & 1 & 1 & \ldots & 0 & 0 & 0 \\
0 & 0 & 1 & 1 & 1 & \ldots & 0 & 0 & 0 \\
\vdots & \vdots & \vdots & \vdots & \vdots & \ddots & \vdots & \vdots & \vdots \\
0 & 0 & 0 & 0 & 0 & \ldots & 1 & 1 & 0 \\
0 & 0 & 0 & 0 & 0 & \ldots & 1 & 1 & 1 \\
0 & 0 & 0 & 0 & 0 & \ldots & 0 & 1 & 1
\end{array}\right) .
$$

If $n=2 m+1$,

$$
M=\sum_{j=1}^{m} \sum_{i=1}^{2 j} e_{i, 2 j+2}+\sum_{j=1}^{m} \sum_{i=1}^{2 j} e_{i, 2 j+3}+\sum_{j=1}^{m} \sum_{i=1}^{2 j+1} e_{i, 2 j-1}+\sum_{j=1}^{m} \sum_{i=1}^{2 j+1} e_{i, 2 j}+\sum_{i=1}^{2 m} e_{i, 2 m+1} .
$$

Combining the third and fifth terms, we get

$$
\begin{aligned}
M= & \sum_{j=1}^{m} \sum_{i=1}^{2 j} e_{i, 2 j+2}+\sum_{j=1}^{m} \sum_{i=1}^{2 j} e_{i, 2 j+3}+\sum_{j=1}^{m+1} \sum_{i=1}^{2 j+1} e_{i, 2 j-1} \\
& +\sum_{j=1}^{m} \sum_{i=1}^{2 j+1} e_{i, 2 j}-e_{m+1, m+1} \\
= & e_{m+1, m+1}+\sum_{k \text { even }}\left(\sum_{i=1}^{k-2} e_{i, k}+\sum_{i=1}^{k+1} e_{i, k}\right)+\sum_{k \text { odd }}\left(\sum_{i=1}^{k-3} e_{i, k}+\sum_{i=1}^{k+2} e_{i, k}\right) \\
= & e_{m+1, m+1}+\sum_{k \text { even }} \sum_{i=k-1}^{k+1} e_{i, k}+\sum_{k \text { odd }} \sum_{i=k-2}^{k+2} e_{i, k} .
\end{aligned}
$$

The right-hand side is again symmetric; it looks like

$$
M_{2 m+1}=\left(\begin{array}{ccccccccc}
1 & 1 & 1 & 0 & 0 & \ldots & 0 & 0 & 0 \\
1 & 1 & 1 & 0 & 0 & \ldots & 0 & 0 & 0 \\
1 & 1 & 1 & 1 & 1 & \ldots & 0 & 0 & 0 \\
0 & 0 & 1 & 1 & 1 & \ldots & 0 & 0 & 0 \\
0 & 0 & 1 & 1 & 1 & \ldots & 0 & 0 & 0 \\
\vdots & \vdots & \vdots & \vdots & \vdots & \ddots & \vdots & \vdots & \vdots \\
0 & 0 & 0 & 0 & 0 & \ldots & 1 & 1 & 1 \\
0 & 0 & 0 & 0 & 0 & \ldots & 1 & 1 & 1 \\
0 & 0 & 0 & 0 & 0 & \ldots & 1 & 1 & 0
\end{array}\right) .
$$

To finish the proof, it remains only to show that the matrices constructed in this way can be lifted to $Z_{2}$ in such a way that the determinant is in $2 Z_{2}^{*}$. The Hensel's lemma argument described above works as long as at least one of the diagonal cofactors $A_{i, i}$ is nonzero. It is easy to prove by induction on $n$ that the cofactor $A_{1,1} \neq 0$. All entries of $M_{2}$, and hence all cofactors, are 1. If $n=2 m>2$, subtract row $2 m$ from row $2 m-1$, then row $2 m-2$ from row $2 m$, and then add row $2 m-1$ to row $2 m$. If $n=2 m+1$, subtract row $2 m$ from row $2 m+1$, add row $2 m+1$ to row $2 m$, then add row $2 m+1$ to row 
$2 m-1$. In each case, the row operations leave the first row untouched and replace $M_{n}$ by the block diagonal matrix

$$
\left(\begin{array}{cc}
M_{n-1} & 0 \\
0 & (-1)^{n}
\end{array}\right) .
$$

In fact, not only does this show that the lower left $n \times n$ submatrix of $M_{n}$ is invertible over $\mathbf{Z} / 2 \mathbf{Z}$, it even proves invertibility over $\mathbf{Z}$. As the first two rows of $M_{n}$ are the same, the matrix $X=2 e_{1,1}+M_{n}$ is a symmetric $n \times n$ matrix such that $X^{n} / 2 \in \mathrm{GL}_{n}(\mathbf{Z})$.

\section{REFERENCES}

[CN] C.-L. Chai and P. Norman, Bad reduction of Seigel moduli schemes with $\Gamma_{0}(p)$-level structure, Amer. J. Math. 112 (1990), 1003-1071.

[G] A. Grothendieck, Groupes de Barsotti-Tate et cristaux de Dieudonné, Séminaire de Mathématiques Supérieures, Presses de l'Université de Montréal, Montréal, 1974.

[Ka] N. Katz, Slope filtration of F-crystals, Asterisque, vol. 63, Soc. Math. France, Paris, 1979. pp. 113-164.

[Ko] N. Koblitz, p-adic variation of the zeta-function over families of varieties defined over the finite fields, Compositio Math. 31 (1975), 119-218.

[La] S. Lang, Algebraic groups over finite fields, Amer. J. Math. 78 (1956), 555-563.

[No] P. Norman, An algorithm for computing local moduli of abelian varieties, Ann. of Math. (2) 101 (1975), 499-509.

[NO] P. Norman and F. Oort, Moduli of abelian varieties, Ann. of Math. (2) 112 (1980), 413-439.

Department of Mathematics, University of Pennsylvania, Philadelphia, PennsylvaNIA 19104

E-mail address, C.-L.Chai: chai@math.upenn.edu

E-mail address, M. Larsen: larsen@math.upenn.edu 